Acta Crystallographica Section E

\section{Structure Reports}

Online

ISSN 1600-5368

\section{Afroza Banu and G. M. Golzar Hossain*}

School of Chemistry, Cardiff University, Cardiff CF10 3AT, Wales

Correspondence e-mail: acsbd@yahoo.com

\section{Key indicators}

Single-crystal X-ray study

$T=150 \mathrm{~K}$

Mean $\sigma(\mathrm{C}-\mathrm{C})=0.004 \AA$

$R$ factor $=0.035$

$w R$ factor $=0.092$

Data-to-parameter ratio $=8.4$

For details of how these key indicators were automatically derived from the article, see http://journals.iucr.org/e.

\title{
A new polymorph of sulfanilic acid monohydrate
}

An orthorhombic polymorph of sulfanilic acid monohydrate, $\mathrm{C}_{6} \mathrm{H}_{7} \mathrm{NO}_{3} \mathrm{~S} \cdot \mathrm{H}_{2} \mathrm{O}$, is described in which there are significant hydrogen-bonding interactions between the components of the structure.

\section{Comment}

The crystal structure of a monoclinic form $\left(P 2_{1} / n\right)$ of sulfanilic acid monohydrate, (II), has been described (Rae \& Maslen, 1962). Here, the structure of an orthorhombic form, (I) $\left(P 2_{1} 2_{1} 2_{1}\right)$, obtained by recrystallization from a methanol solution of the compound, is described (Fig. 1 and Table 1).<smiles>[NH2+][NH2+]c1ccc(S(=O)(=O)[O-])cc1</smiles>

(I)

The $\mathrm{C}-\mathrm{S}$ and $\mathrm{C}-\mathrm{N}$ bond lengths in (I) (Table 1) are close to the corresponding distances in (II) and $\mathrm{O}_{3} \mathrm{SC}_{6} \mathrm{H}_{4} \mathrm{NH}-\mathrm{CH}-$ $\mathrm{N}\left(\mathrm{CH}_{3}\right)_{2} \cdot \mathrm{H}_{2} \mathrm{O}$ (Hempel et al., 1999). The $\mathrm{S}-\mathrm{O}$ bond distances in (I) are similar to those found in (II) (Rae \& Maslen, 1962), in metanilic acid (Hall \& Maslen, 1965), and in 2,5-dichlorobenzenesulfonic acid and 2,5-dibromobenzenesulfonic acid (Lundgren \& Lundin, 1972). The $\mathrm{C}-\mathrm{S}-\mathrm{O}$ and $\mathrm{O}-\mathrm{S}-\mathrm{O}$ angles deviate from $109.5^{\circ}$ in the expected manner.

The crystal structure of (I) is stabilized by intermolecular $\mathrm{N}-\mathrm{H} \cdots \mathrm{O}$ and $\mathrm{O}-\mathrm{H} \cdots \mathrm{O}$ hydrogen bonds (Table 2), which result in the formation of a hydrogen-bonded network (Fig. 2). The water molecule is hydrogen bonded to the amine group $(\mathrm{N} 1 / \mathrm{H} 1 B)$. The distance between the two parallel structures, with symmetry $(1+x, y, z)$, in the packing diagram (Fig. 2$)$ is 6.163 (3) A.

\section{Experimental}

Sulfanilic acid $(1.732 \mathrm{~g}, 1 \mathrm{mmol})$ was dissolved in methanol $(20 \mathrm{ml})$ and stirred for $1 \mathrm{~h}$. After filtration, the clear solution was left for crystallization, and after two weeks, pale-yellow crystals were obtained.

Crystal data

$\mathrm{C}_{6} \mathrm{H}_{7} \mathrm{NO}_{3} \mathrm{~S} \cdot \mathrm{H}_{2} \mathrm{O}$
$M_{r}=191.20$
Orthorhombic, $P 2_{1} 2_{1} 2_{1}$
$a=6.1630(6) \AA$
$b=6.9607(5) \AA$
$c=18.3251(10) \AA$
$V=786.12(10) \AA^{3}$

$Z=4$

$D_{x}=1.616 \mathrm{Mg} \mathrm{m}^{-3}$

Mo $K \alpha$ radiation

$\mu=0.39 \mathrm{~mm}^{-1}$

$T=150(2) \mathrm{K}$

Block, pale yellow

$0.25 \times 0.22 \times 0.20 \mathrm{~mm}$
Received 29 March 2006

Accepted 1 May 2006 


\section{Data collection}

Enraf-Nonius CAD-4

$\omega / \theta$ scans

Absorption correction: part of the refinement model $(\Delta F)$

(Walker \& Stuart, 1983)

$T_{\min }=0.910, T_{\max }=0.927$

1822 measured reflections

\section{Refinement}

Refinement on $F^{2}$

$R\left[F^{2}>2 \sigma\left(F^{2}\right)\right]=0.035$

$w R\left(F^{2}\right)=0.092$

$S=1.04$

957 reflections

114 parameters

$\mathrm{H}$ atoms treated by a mixture of

independent and constrained

refinement

Table 1

Selected geometric parameters $\left(\AA{ }^{\circ}\right)$.

\begin{tabular}{lrlr}
\hline $\mathrm{S} 1-\mathrm{O} 1$ & $1.448(3)$ & $\mathrm{S} 1-\mathrm{C} 1$ & $1.773(3)$ \\
$\mathrm{S} 1-\mathrm{O} 2$ & $1.459(3)$ & $\mathrm{N} 1-\mathrm{C} 4$ & $1.468(4)$ \\
$\mathrm{S} 1-\mathrm{O} 3$ & $1.446(3)$ & & \\
$\mathrm{O} 1-\mathrm{S} 1-\mathrm{O} 2$ & $111.64(19)$ & $\mathrm{O} 2-\mathrm{S} 1-\mathrm{O} 3$ & $112.15(15)$ \\
$\mathrm{O} 1-\mathrm{S} 1-\mathrm{O} 3$ & $113.77(19)$ & $\mathrm{O} 2-\mathrm{S} 1-\mathrm{C} 1$ & $105.00(15)$ \\
$\mathrm{O} 1-\mathrm{S} 1-\mathrm{C} 1$ & $106.26(15)$ & $\mathrm{O} 3-\mathrm{S} 1-\mathrm{C} 1$ & $107.36(15)$ \\
\hline
\end{tabular}

Table 2

Hydrogen-bond geometry $\left(\AA,^{\circ}\right)$.

\begin{tabular}{|c|c|c|c|c|}
\hline$D-\mathrm{H} \cdots A$ & $D-\mathrm{H}$ & $\mathrm{H} \cdots A$ & $D \cdots A$ & $D-\mathrm{H} \cdots A$ \\
\hline $\mathrm{O} 4-\mathrm{H} 4 A \cdots \mathrm{O} 1^{\mathrm{i}}$ & 0.95 & 1.90 & $2.821(3)$ & 163 \\
\hline $\mathrm{O} 4-\mathrm{H} 4 B \cdots \mathrm{O} 2^{\mathrm{ii}}$ & 0.95 & 1.89 & $2.838(4)$ & 175 \\
\hline $\mathrm{N} 1-\mathrm{H} 1 A \cdots \mathrm{O} 3^{\mathrm{iii}}$ & 0.94 & 1.97 & $2.846(4)$ & 154 \\
\hline $\mathrm{N} 1-\mathrm{H} 1 B \cdots \mathrm{O} 4$ & 0.93 & 1.84 & $2.738(3)$ & 160 \\
\hline $\mathrm{N} 1-\mathrm{H} 1 C \cdots \mathrm{O} 2^{\mathrm{iv}}$ & 0.96 & 1.95 & $2.895(4)$ & 166 \\
\hline
\end{tabular}

In the absence of significant anomalous scattering, Friedel pairs were merged before the final refinement. $\mathrm{C}$-bound $\mathrm{H}$ atoms were included in the riding model approximation with $\mathrm{C}-\mathrm{H}=0.95 \AA$, and with $U_{\text {iso }}(\mathrm{H})=1.2 U_{\text {eq }}(\mathrm{C}) . \mathrm{H}$ atoms attached to $\mathrm{N}$ and $\mathrm{O}$ (water) were located from an electron density map, fixed in these positions and assigned individual isotropic displacement parameters; see Table 2 for bond distances.

Data collection: CAD-4 EXPRESS (Enraf-Nonius, 1992); cell refinement: CAD-4 EXPRESS; data reduction: CAD-4 Processing Program (Hursthouse, 1976); program(s) used to solve structure: SHELXS97 (Sheldrick, 1990); program(s) used to refine structure: SHELXL97 (Sheldrick, 1997); molecular graphics: ORTEP-3 for

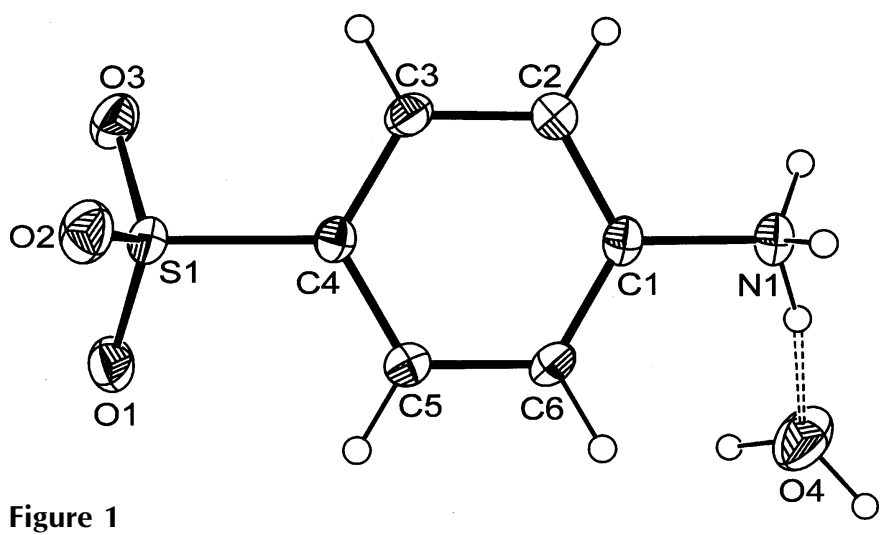

The asymmetric unit of (I), showing the atom-labelling scheme. Displacement ellipsoids are drawn at the $35 \%$ probability level. The hydrogen bond is shown as a dashed line.

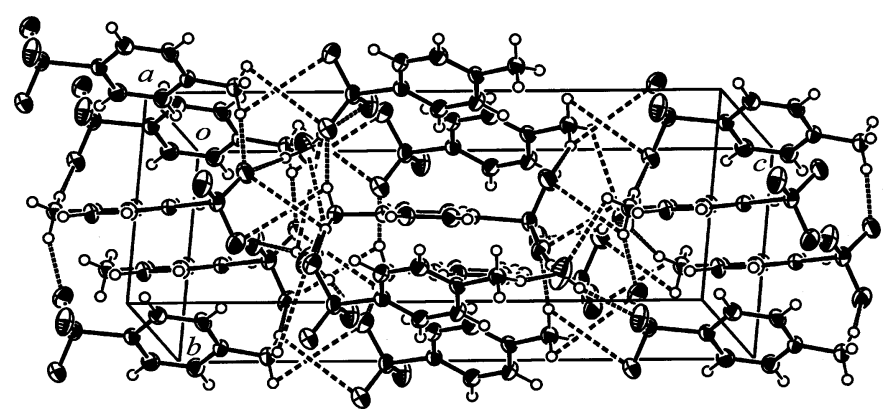

Figure 2

The molecular packing of (I), viewed approximately along the $a$ axis. Dashed lines indicate the hydrogen-bonding interactions.

Windows (Farrugia, 1997); software used to prepare material for publication: Win $G X$ (Farrugia, 1999).

GMGH acknowledges the Ministry of Science and Technology, The People's Republic of Bangladesh, for the award of a Bangabandhu Fellowship.

\section{References}

Enraf-Nonius (1992). CAD-4 EXPRESS. Enraf-Nonius, Delft, The Netherlands.

Farrugia, L. J. (1997). J. Appl. Cryst. 30, 565.

Farrugia, L. J. (1999). J. Appl. Cryst. 32, 837-838.

Hall, S. R. \& Maslen, E. N. (1965). Acta Cryst. 18, 301-306.

Hempel, A., Camerman, N., Mastropaolo, D. \& Camerman, A. (1999). Acta Cryst. C55, 697-698.

Hursthouse, M. B. (1976). CAD-4 Processing Program. Queen Mary College, London.

Lundgren, J.-O. \& Lundin, P. (1972). Acta Cryst. B28, 486-491.

Rae, A. I. M. \& Maslen, E. N. (1962). Acta Cryst. 15, 1285-1291.

Sheldrick, G. M. (1990). Acta Cryst. A46, 467-473.

Sheldrick, G. M. (1997). SHELXL97. University of Gottingen, Germany.

Walker, N. \& Stuart, D. (1983). Acta Cryst. A39, 158-166. 


\title{
supporting information
}

Acta Cryst. (2006). E62, o2252-o2253 [https://doi.org/10.1107/S1600536806016060]

\section{A new polymorph of sulfanilic acid monohydrate}

\author{
Afroza Banu and G. M. Golzar Hossain \\ sulfanilic acid monohydrate \\ Crystal data \\ $\mathrm{C}_{6} \mathrm{H}_{7} \mathrm{NO}_{3} \mathrm{~S} \cdot \mathrm{H}_{2} \mathrm{O}$ \\ $F(000)=400$ \\ $M_{r}=191.20$ \\ Orthorhombic, $P 2_{1} 2_{1} 2_{1}$ \\ Hall symbol: P $2 \mathrm{ac} 2 \mathrm{ab}$ \\ $a=6.1630$ (6) $\AA$ \\ $b=6.9607(5) \AA$ \\ $c=18.3251(10) \AA$ \\ $V=786.12(10) \AA^{3}$ \\ $Z=4$ \\ $D_{\mathrm{x}}=1.616 \mathrm{Mg} \mathrm{m}^{-3}$ \\ Mo $K \alpha$ radiation, $\lambda=0.71073 \AA$ \\ Cell parameters from 957 reflections \\ $\theta=2.9-26.3^{\circ}$ \\ $\mu=0.39 \mathrm{~mm}^{-1}$ \\ $T=150 \mathrm{~K}$ \\ Block, pale yellow \\ $0.25 \times 0.22 \times 0.20 \mathrm{~mm}$
}

\section{Data collection}

Enraf-Nonius CAD-4 diffractometer

Radiation source: fine-focus sealed tube

Graphite monochromator

$\omega / \theta$ scans

Absorption correction: part of the refinement model $(\Delta F)$

(Walker \& Stuart, 1983)

$T_{\min }=0.910, T_{\max }=0.927$

3 measured reflections

\section{Refinement}

Refinement on $F^{2}$

Least-squares matrix: full

$R\left[F^{2}>2 \sigma\left(F^{2}\right)\right]=0.035$

$w R\left(F^{2}\right)=0.092$

$S=1.04$

957 reflections

114 parameters

5 restraints

Primary atom site location: structure-invariant direct methods

957 independent reflections

793 reflections with $I>2 \sigma(I)$

$R_{\text {int }}=0.024$

$\theta_{\max }=26.3^{\circ}, \theta_{\min }=2.2^{\circ}$

$h=-7 \rightarrow 0$

$k=-8 \rightarrow 2$

$l=-22 \rightarrow 22$

1534 standard reflections every 134 reflections intensity decay: none

Secondary atom site location: difference Fourier map

Hydrogen site location: inferred from neighbouring sites

$\mathrm{H}$ atoms treated by a mixture of independent and constrained refinement

$w=1 /\left[\sigma^{2}\left(F_{\mathrm{o}}^{2}\right)+(0.0544 P)^{2}+0.1313 P\right]$ where $P=\left(F_{\mathrm{o}}^{2}+2 F_{\mathrm{c}}^{2}\right) / 3$

$(\Delta / \sigma)_{\max }=0.001$

$\Delta \rho_{\max }=0.31 \mathrm{e} \AA^{-3}$

$\Delta \rho_{\text {min }}=-0.29$ e $\AA^{-3}$

Absolute structure: Flack (1983) 


\section{Special details}

Geometry. All e.s.d.'s (except the e.s.d. in the dihedral angle between two 1.s. planes) are estimated using the full covariance matrix. The cell e.s.d.'s are taken into account individually in the estimation of e.s.d.'s in distances, angles and torsion angles; correlations between e.s.d.'s in cell parameters are only used when they are defined by crystal symmetry. An approximate (isotropic) treatment of cell e.s.d.'s is used for estimating e.s.d.'s involving 1.s. planes.

Refinement. Refinement of $F^{2}$ against ALL reflections. The weighted $R$-factor $w R$ and goodness of fit $S$ are based on $F^{2}$, conventional $R$-factors $R$ are based on $F$, with $F$ set to zero for negative $F^{2}$. The threshold expression of $F^{2}>\sigma\left(F^{2}\right)$ is used only for calculating $R$-factors (gt) etc. and is not relevant to the choice of reflections for refinement. $R$-factors based on $F^{2}$ are statistically about twice as large as those based on $F$, and $R$ - factors based on ALL data will be even larger.

Fractional atomic coordinates and isotropic or equivalent isotropic displacement parameters $\left(\hat{A}^{2}\right)$

\begin{tabular}{lllll}
\hline & $x$ & $y$ & $z$ & $U_{\text {iso }} * / U_{\text {eq }}$ \\
\hline S1 & $-0.01053(14)$ & $0.48483(12)$ & $0.63841(4)$ & $0.0291(2)$ \\
O1 & $0.2228(4)$ & $0.5066(5)$ & $0.63447(13)$ & $0.0463(7)$ \\
O2 & $-0.1130(5)$ & $0.6505(4)$ & $0.67294(13)$ & $0.0386(7)$ \\
O3 & $-0.0795(5)$ & $0.3055(4)$ & $0.67078(13)$ & $0.0428(7)$ \\
O4 & $0.0515(4)$ & $0.6429(5)$ & $0.25576(15)$ & $0.0663(11)$ \\
N1 & $-0.3138(4)$ & $0.5290(4)$ & $0.32896(13)$ & $0.0307(7)$ \\
C1 & $-0.1055(5)$ & $0.4873(4)$ & $0.54710(15)$ & $0.0252(7)$ \\
C2 & $-0.3124(6)$ & $0.5482(5)$ & $0.53154(17)$ & $0.0303(8)$ \\
H2 & -0.4084 & 0.5827 & 0.5699 & $0.036^{*}$ \\
C3 & $-0.3807(6)$ & $0.5591(5)$ & $0.45936(17)$ & $0.0307(8)$ \\
H3 & -0.5234 & 0.6011 & 0.4480 & $0.037^{*}$ \\
C4 & $-0.2392(5)$ & $0.5081(5)$ & $0.40465(16)$ & $0.0273(7)$ \\
C5 & $-0.0359(6)$ & $0.4401(5)$ & $0.41925(16)$ & $0.0304(8)$ \\
H5 & 0.0554 & 0.4006 & 0.3803 & $0.036^{*}$ \\
C6 & $0.0381(4)$ & $0.4282(4)$ & $0.49055(13)$ & $0.0299(8)$ \\
H6 & 0.1797 & 0.3823 & 0.5013 & $0.036^{*}$ \\
H1A & -0.3781 & 0.4113 & 0.3158 & $0.053(13)^{*}$ \\
H1B & -0.1976 & 0.5436 & 0.2970 & $0.041(11)^{*}$ \\
H1C & -0.4142 & 0.6317 & 0.3197 & $0.059(14)^{*}$ \\
H4A & 0.1434 & 0.6163 & 0.2156 & $0.12(2)^{*}$ \\
H4B & 0.1615 & 0.7184 & 0.2777 & $0.15(3)^{*}$ \\
& & & &
\end{tabular}

Atomic displacement parameters $\left(\AA^{2}\right)$

\begin{tabular}{lllllll}
\hline & $U^{11}$ & $U^{22}$ & $U^{33}$ & $U^{12}$ & $U^{13}$ & $U^{23}$ \\
\hline S1 & $0.0329(4)$ & $0.0347(4)$ & $0.0195(3)$ & $0.0022(5)$ & $-0.0003(3)$ & $0.0014(4)$ \\
O1 & $0.0293(13)$ & $0.079(2)$ & $0.0311(12)$ & $-0.0029(16)$ & $-0.0032(10)$ & $0.0047(17)$ \\
O2 & $0.0500(18)$ & $0.0387(13)$ & $0.0270(12)$ & $0.0017(14)$ & $0.0012(14)$ & $-0.0070(11)$ \\
O3 & $0.0549(19)$ & $0.0419(14)$ & $0.0315(13)$ & $0.0020(14)$ & $0.0023(15)$ & $0.0098(11)$ \\
O4 & $0.052(2)$ & $0.096(3)$ & $0.0513(16)$ & $-0.025(2)$ & $0.0144(17)$ & $-0.0228(18)$ \\
N1 & $0.0376(16)$ & $0.0327(16)$ & $0.0217(12)$ & $-0.0033(15)$ & $-0.0041(13)$ & $0.0000(12)$ \\
C1 & $0.0299(16)$ & $0.0241(15)$ & $0.0216(13)$ & $-0.0018(18)$ & $0.0004(12)$ & $0.0007(13)$ \\
C2 & $0.0306(18)$ & $0.0334(18)$ & $0.0268(16)$ & $0.0028(16)$ & $0.0057(14)$ & $-0.0008(14)$ \\
C3 & $0.0296(19)$ & $0.0331(17)$ & $0.0294(16)$ & $0.0016(16)$ & $-0.0019(14)$ & $-0.0002(14)$ \\
C4 & $0.0353(17)$ & $0.0254(16)$ & $0.0212(15)$ & $0.0001(17)$ & $-0.0030(12)$ & $-0.0001(15)$ \\
C5 & $0.0321(19)$ & $0.0353(17)$ & $0.0237(14)$ & $0.0036(15)$ & $0.0054(15)$ & $-0.0067(13)$
\end{tabular}




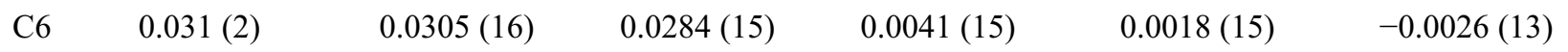

Geometric parameters $(\AA, \stackrel{o}{)}$

\begin{tabular}{|c|c|c|c|}
\hline $\mathrm{S} 1-\mathrm{O} 1$ & $1.448(3)$ & $\mathrm{C} 1-\mathrm{C} 2$ & $1.374(5)$ \\
\hline $\mathrm{S} 1-\mathrm{O} 2$ & $1.459(3)$ & $\mathrm{C} 1-\mathrm{C} 6$ & $1.424(4)$ \\
\hline $\mathrm{S} 1-\mathrm{O} 3$ & $1.446(3)$ & $\mathrm{C} 2-\mathrm{C} 3$ & $1.390(4)$ \\
\hline $\mathrm{S} 1-\mathrm{C} 1$ & $1.773(3)$ & $\mathrm{C} 2-\mathrm{H} 2$ & 0.9500 \\
\hline $\mathrm{O} 4-\mathrm{H} 4 \mathrm{~A}$ & 0.9476 & $\mathrm{C} 3-\mathrm{C} 4$ & $1.376(5)$ \\
\hline $\mathrm{O} 4-\mathrm{H} 4 \mathrm{~B}$ & 0.9474 & $\mathrm{C} 3-\mathrm{H} 3$ & 0.9500 \\
\hline $\mathrm{N} 1-\mathrm{C} 4$ & $1.468(4)$ & $\mathrm{C} 4-\mathrm{C} 5$ & $1.366(5)$ \\
\hline $\mathrm{N} 1-\mathrm{H} 1 \mathrm{~A}$ & 0.9415 & $\mathrm{C} 5-\mathrm{C} 6$ & $1.386(4)$ \\
\hline N1-H1B & 0.9315 & $\mathrm{C} 5-\mathrm{H} 5$ & 0.9500 \\
\hline $\mathrm{N} 1-\mathrm{H} 1 \mathrm{C}$ & 0.9606 & C6- 6 6 & 0.9500 \\
\hline $\mathrm{O} 1-\mathrm{S} 1-\mathrm{O} 2$ & 111.64 (19) & $\mathrm{C} 1-\mathrm{C} 2-\mathrm{C} 3$ & $119.7(3)$ \\
\hline $\mathrm{O} 1-\mathrm{S} 1-\mathrm{O} 3$ & 113.77 (19) & $\mathrm{C} 1-\mathrm{C} 2-\mathrm{H} 2$ & 120.2 \\
\hline $\mathrm{O} 1-\mathrm{S} 1-\mathrm{C} 1$ & $106.26(15)$ & $\mathrm{C} 3-\mathrm{C} 2-\mathrm{H} 2$ & 120.2 \\
\hline $\mathrm{O} 2-\mathrm{S} 1-\mathrm{O} 3$ & $112.15(15)$ & $\mathrm{C} 4-\mathrm{C} 3-\mathrm{C} 2$ & $119.2(3)$ \\
\hline $\mathrm{O} 2-\mathrm{S} 1-\mathrm{C} 1$ & $105.00(15)$ & $\mathrm{C} 4-\mathrm{C} 3-\mathrm{H} 3$ & 120.4 \\
\hline $\mathrm{O} 3-\mathrm{S} 1-\mathrm{C} 1$ & $107.36(15)$ & $\mathrm{C} 2-\mathrm{C} 3-\mathrm{H} 3$ & 120.4 \\
\hline $\mathrm{H} 4 \mathrm{~A}-\mathrm{O} 4-\mathrm{H} 4 \mathrm{~B}$ & 90.5 & $\mathrm{C} 5-\mathrm{C} 4-\mathrm{C} 3$ & $121.9(3)$ \\
\hline $\mathrm{C} 4-\mathrm{N} 1-\mathrm{H} 1 \mathrm{~A}$ & 106.8 & $\mathrm{C} 5-\mathrm{C} 4-\mathrm{N} 1$ & $120.4(3)$ \\
\hline $\mathrm{C} 4-\mathrm{N} 1-\mathrm{H} 1 \mathrm{~B}$ & 111.4 & $\mathrm{C} 3-\mathrm{C} 4-\mathrm{N} 1$ & $117.7(3)$ \\
\hline $\mathrm{H} 1 \mathrm{~A}-\mathrm{N} 1-\mathrm{H} 1 \mathrm{~B}$ & 104.9 & $\mathrm{C} 4-\mathrm{C} 5-\mathrm{C} 6$ & 120.5 \\
\hline $\mathrm{C} 4-\mathrm{N} 1-\mathrm{H} 1 \mathrm{C}$ & 116.3 & $\mathrm{C} 4-\mathrm{C} 5-\mathrm{H} 5$ & 119.8 \\
\hline $\mathrm{H} 1 \mathrm{~A}-\mathrm{N} 1-\mathrm{H} 1 \mathrm{C}$ & 109.3 & $\mathrm{C} 6-\mathrm{C} 5-\mathrm{H} 5$ & 119.8 \\
\hline $\mathrm{H} 1 \mathrm{~B}-\mathrm{N} 1-\mathrm{H} 1 \mathrm{C}$ & 107.6 & $\mathrm{C} 5-\mathrm{C} 6-\mathrm{C} 1$ & $117.7(3)$ \\
\hline $\mathrm{C} 2-\mathrm{C} 1-\mathrm{C} 6$ & $121.0(3)$ & $\mathrm{C} 5-\mathrm{C} 6-\mathrm{H} 6$ & 121.2 \\
\hline $\mathrm{C} 2-\mathrm{C} 1-\mathrm{S} 1$ & $120.4(2)$ & $\mathrm{C} 1-\mathrm{C} 6-\mathrm{H} 6$ & 121.2 \\
\hline $\mathrm{C} 6-\mathrm{C} 1-\mathrm{S} 1$ & $118.6(2)$ & & \\
\hline
\end{tabular}

Hydrogen-bond geometry $\left(A,{ }^{\circ}\right)$

\begin{tabular}{lllll}
\hline$D-\mathrm{H} \cdots A$ & $D-\mathrm{H}$ & $\mathrm{H} \cdots A$ & $D \cdots A$ & $D-\mathrm{H} \cdots A$ \\
\hline $\mathrm{O} 4-\mathrm{H} 4 \mathrm{a} \cdots \mathrm{O} 1^{\mathrm{i}}$ & 0.95 & 1.90 & $2.821(3)$ & 163 \\
$\mathrm{O} 4-\mathrm{H} 4 \mathrm{~b} \cdots \mathrm{O} 2^{\mathrm{ii}}$ & 0.95 & 1.89 & $2.838(4)$ & 175 \\
$\mathrm{~N} 1-\mathrm{H} 1 \mathrm{a} \cdots \mathrm{O} 3^{i i i}$ & 0.94 & 1.97 & $2.846(4)$ & 154 \\
$\mathrm{~N} 1-\mathrm{H} 1 \mathrm{~b} \cdots \mathrm{O} 4$ & 0.93 & 1.84 & $2.738(3)$ & 160 \\
$\mathrm{~N} 1-\mathrm{H} 1 \mathrm{c} \cdots \mathrm{O} 2^{\text {iv }}$ & 0.96 & 1.95 & $2.895(4)$ & 166
\end{tabular}

Symmetry codes: (i) $-x+1 / 2,-y+1, z-1 / 2$; (ii) $x+1 / 2,-y+3 / 2,-z+1$; (iii) $x-1 / 2,-y+1 / 2,-z+1$; (iv) $x-1 / 2,-y+3 / 2,-z+1$. 